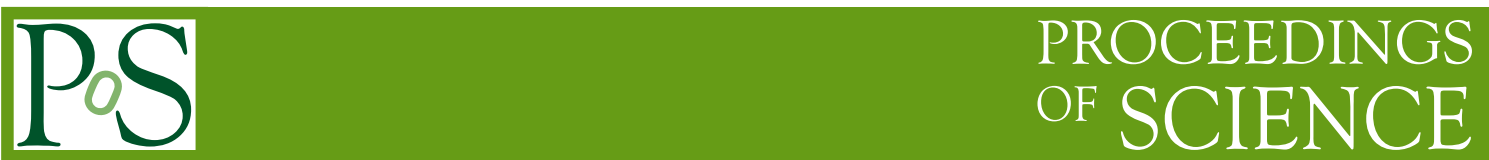

\title{
Simulations of MPD Straw End-Cap Tracker
}

\author{
Ján Fedorišin* \\ Veksler and Baldin Laboratory of High Energy Physics, Joint Institute for Nuclear Research \\ E-mail: fedorisinejinr.ru
}

A response of the MPD Straw End-Cap Tracker has been estimated employing FAIRROOT, GEANT3, GARFIELD and the hit simulation programs. The MC simulations allowed us to study the following detector characteristics: the occupancy, the charge clusters distributions and energy losses, drift properties (electron and ion drift times, electron attachment probabilities, drift and avalanche regions), gas gain, anode signals, the integrated anode charges. The calibration method [1], [2], [3] has been used to estimate the hit distance of closest approach (DCA) coordinates that are necessary for the track reconstruction. The DCA coordinate resolution varies from $100 \mu \mathrm{m}$ to $300 \mu \mathrm{m}$ depending on the radial distance from anode wire. Our results are found compatible with the results from other HEP experiments employing straw tube detectors, e.g. PANDA [1] and ATLAS [2], [3].

XXII International Baldin Seminar on High Energy Physics Problems,

15-20 September 2014

JINR, Dubna, Russia

\footnotetext{
${ }^{*}$ Speaker.
} 


\section{Introduction}

Multi-Purpose Detector (MPD) should provide an identification of charged particles produced in heavy-ion collisions at NICA collider energies, i.e. for $\sqrt{s_{N N}}=4-11 \mathrm{GeV}$ [4]. The detector consists of several detector subsystems providing information on the coordinates and energies of measured particles. The general view of the proposed experimental apparatus is presented in Fig.1. Straw End-Cap Tracker (ECT) is along with the TPC, CPC and TOF detectors included in the MPD tracking system. There are altogether four independent identical Straw ECT modules placed at distances $2 \mathrm{~m}<|z|<3 \mathrm{~m}$ from the origin of the coordinate system and arranged symmetrically along the beam pipe. The low occupancy and high uniformity of the straw tube detector is achieved through its carefully chosen technical design which is described in the next Section.

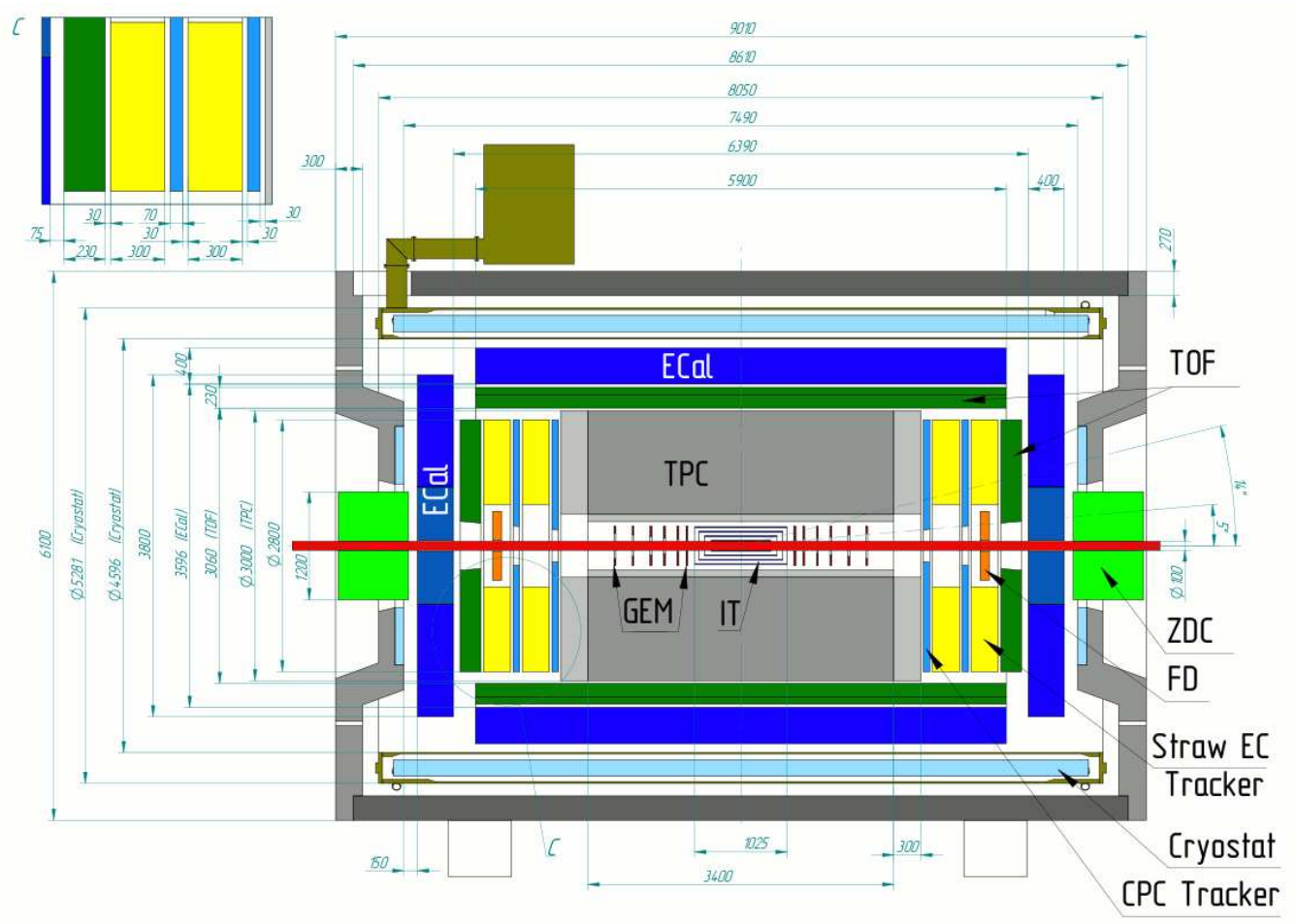

Figure 1: Cutaway side view of the central MPD with basic dimensions expressed in millimeters

\section{Layout of MPD Straw End-Cap Tracker}

Straw End-Cap Tracker has segmented hierarchical structure presented in Fig.2.

Each module is divided into 5 submodules and each submodule contains 6 layers with 600 kapton straw tubes per layer. The longitudinal size of the submodule is $25 \mathrm{~cm}$ with $2.5 \mathrm{~cm}$ added on each side as a reserve space. The originally proposed outer radius of $110 \mathrm{~cm}$ has been in 2012 increased to $140 \mathrm{~cm}$.

There are actually three types of straw tube layers depending on how the tubes are inclined with respect to radial tubes. First layer of the submodule is composed of purely radial tubes, while the 


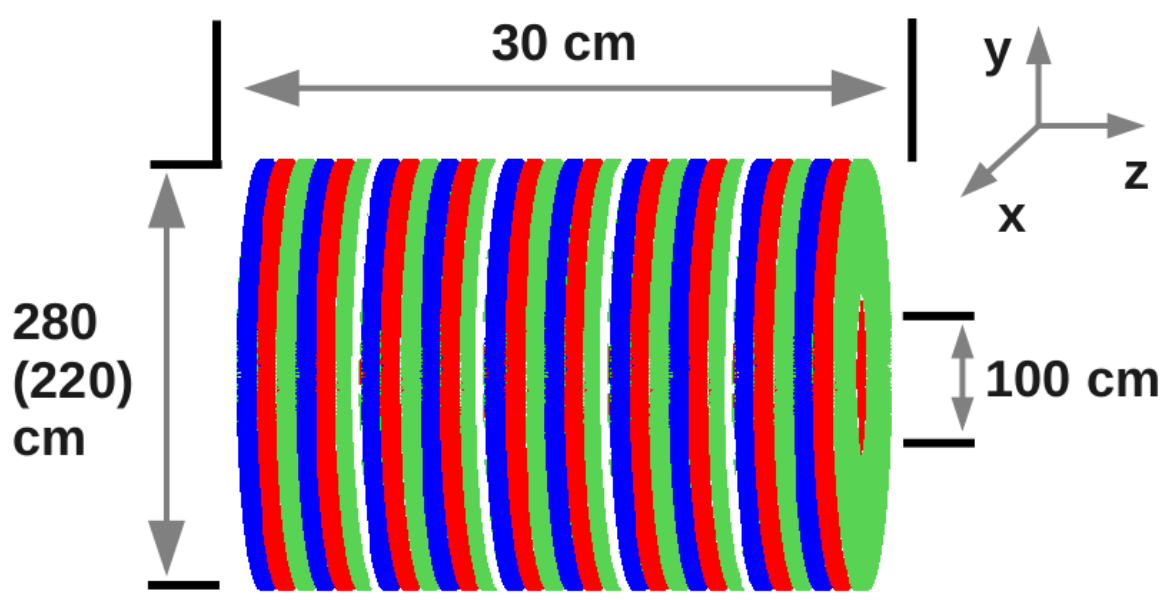

Figure 2: Straw ECT module structure

tubes in the next two layers are inclined by angles $\alpha=+7^{\circ}$ and $\alpha=-7^{\circ}$ respectively. This pattern is repeated for the next three submodule layers as well. Varying the incline angle in consecutive straw layers is necessary to determine the coordinates of detected particles.

The type of layer is reflected in Fig. 2 by the three different colours: blue $\left(\alpha=0^{\circ}\right)$, red $(\alpha=$ $\left.+7^{\circ}\right)$ and green $\left(\alpha=-7^{\circ}\right)$.

The structure and material composition of the straw tube is indicated in Fig.3.

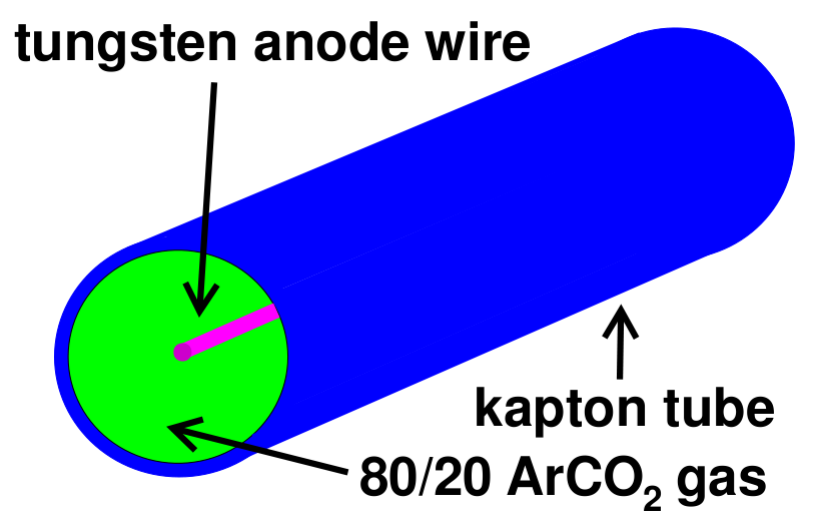

Figure 3: Schematic structure of straw tube

The radius of straw tube $r_{\text {tube }}=2 \mathrm{~mm}$ was chosen as a reasonable compromise between the response speed, the number of detecting channels $\left(72 \times 10^{3}\right)$ and the detector occupancy. The 80/20 $\mathrm{ArCO}_{2}$ gas mixture is assumed to work at room temperature and atmospheric pressure, with anode voltage $V_{A}=1650 \mathrm{~V}$.

\section{Detector occupancy}

The occupancy of the Straw ECT is estimated using GEANT simulations of UrQMD Au+Au 
collisions at $\sqrt{s_{N N}}=9 \mathrm{GeV}$. Fig.4 presents the radial dependence of mean number of tracks per $1 \mathrm{~cm}$ of the straw tube. Integration over the full tube length gives the occupancy per 1 tube which is about $12 \%$ for the used data sample.

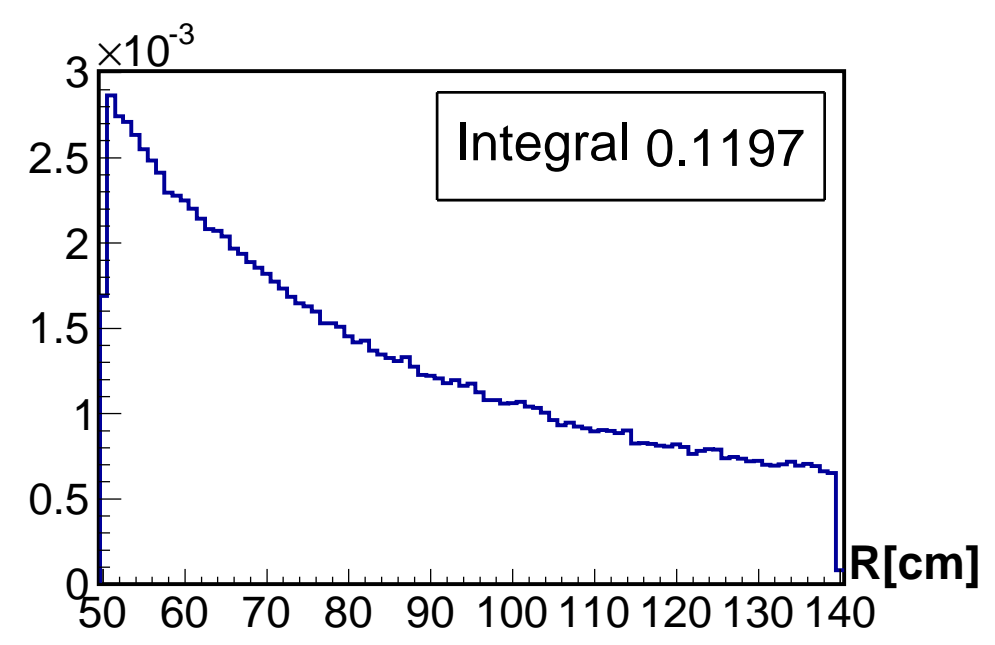

Figure 4: Number of tracks per $1 \mathrm{~cm}$ of straw tubes (the radial layers)

Fig. 5 shows the fraction of multiple hits per the straw tube.

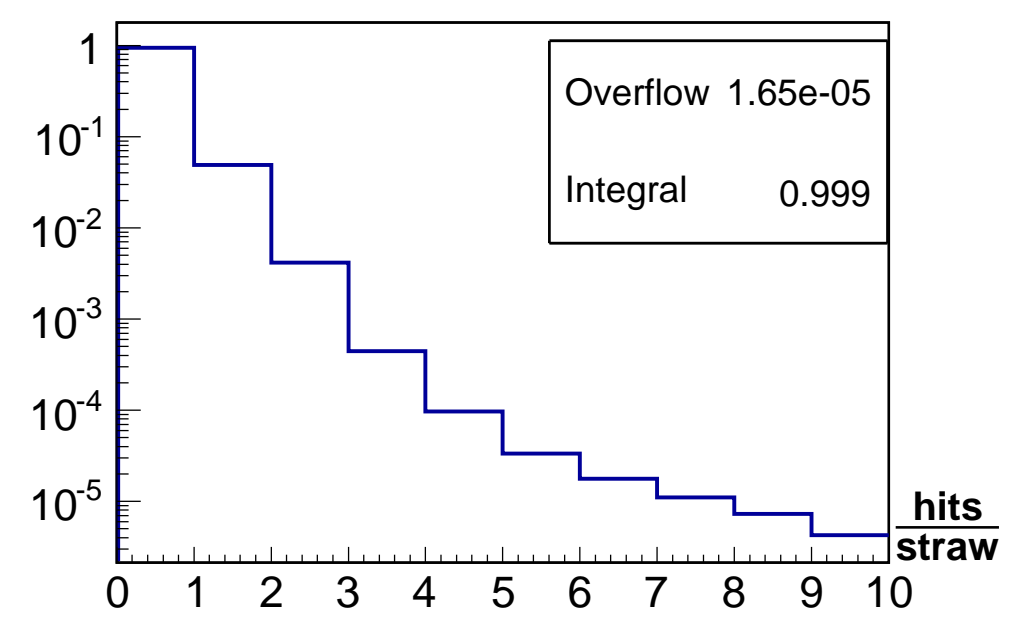

Figure 5: Number of hits per straw tube

Multiple hits occur in approximately $0.5 \%$ of all tubes, i.e. in about 3 tubes (out of 600 ) per straw layer.

\section{Simulations with GARFIELD}

To simulate the detector response in detail, we must include GARFIELD programming package [5], [6] in our studies. The C++ based version of GARFIELD called GARFIELD++ is objectoriented program toolkit for the detailed simulations of particle detectors which use a gas mixture 
or a semiconductor material as sensitive medium. The list of tasks that can be solved employing GARFIELD includes:

- simulations of detector sensitive media;

- simulations of electric and magnetic fields;

- ionization of detector sensitive materials by charged particles;

- charge transport (drift of electrons and ions under the influence of electric and magnetic fields);

- signals induced on electrodes.

Signal simulation in gas-based particle detectors is an exacting, complex task that requires a gradual solution of a few partial subtasks. The subtasks are in general classified in three groups:

1. primary charge generation, i.e. distribution of electron-ion clusters along particle path in active detector volume;

2. drift of primary charge towards the electrodes, avalanche near the anode, gas amplification;

3. anode signal simulation.

The concept is schematically illustrated in Fig.6.

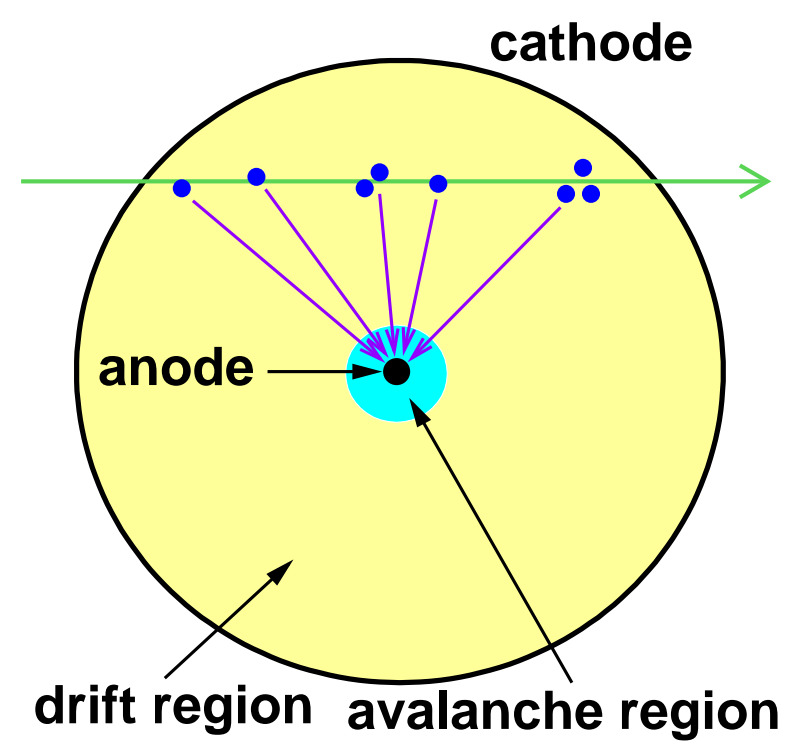

Figure 6: Drift of electrons left by charged particle in straw tube

Subsequent analysis of simulated hits provides necessary information on the coordinates of particle tracks. Additional information about the corresponding energy losses in detector sensitive volumes is helpful to identify the particle species. 


\section{Cluster generation}

Electron/ion clusters produced along a path of charged particle are described by the two basic properties. The first characteristic is a mean number of electron clusters per $\mathrm{cm}$ and the second one is a number of electrons/ions per cluster. Both the characteristics depend on the working gas properties such as its chemical composition, pressure and temperature. They also depend on a particle type and momentum.

Probability to create $k$ clusters on path $d$ inside a tube is described by Poisson distribution:

$$
p_{\lambda}(k)=\lambda^{k} \frac{\exp (-\lambda)}{k !}, \quad \lambda=\rho d,
$$

where $\rho$ is a mean number of clusters per $\mathrm{cm}$. Inverse $1 / \rho$ is thus a mean free path of particle in tube volume.

Spatial distribution of clusters on path $d$ inside a tube is in general obtained as a chain of consecutive exponential probabilities:

$$
\frac{1}{\rho} \exp \left(-l_{i} \rho\right), \quad i=1,2, \ldots
$$

where $l_{1}+l_{2}+\ldots \leq d$. If $1 / \rho \ll d$, the distribution of clusters can be regarded as uniform.

The cluster densities of particles produced in the studied collisions are provided by GARFIELD. They are shown in Fig.7 as functions of particle momentum.

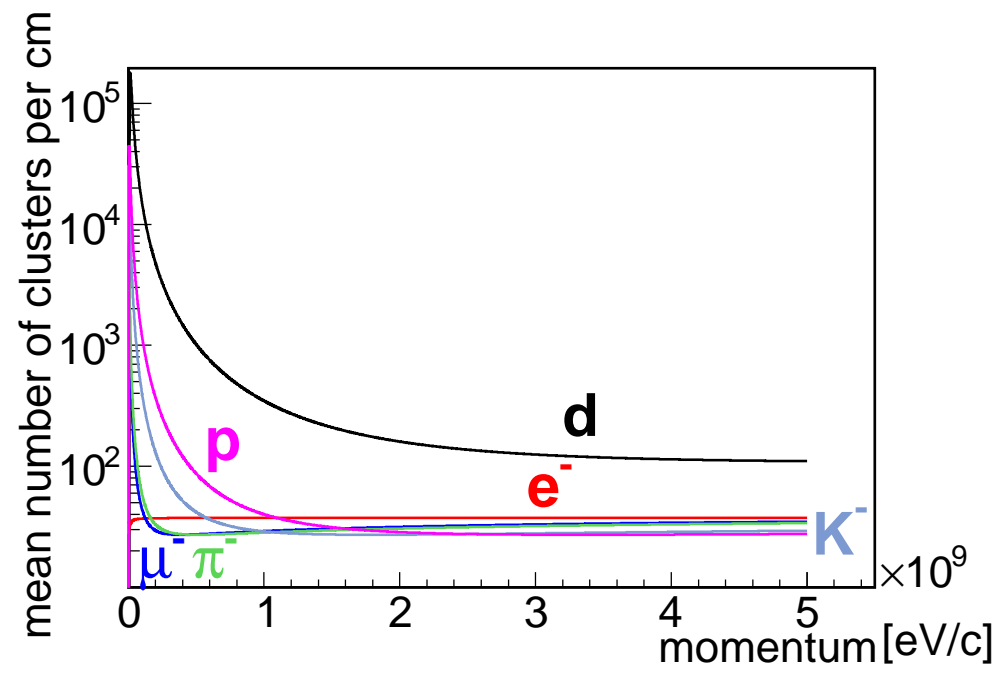

Figure 7: Cluster density vs momentum

The lists of particles produced in the GEANT simulations and particles available in GARFIELD are compared in Tab.1.

\begin{tabular}{|l|l|l|l|l|l|l|l|l|l|l|l|l|l|l|l|l|}
\hline GEANT & $e^{-}$ & $e^{+}$ & $\mu^{-}$ & $\mu^{+}$ & $\pi^{-}$ & $\pi^{+}$ & $K^{-}$ & $K^{+}$ & $p$ & $\bar{p}$ & $\Sigma^{-}$ & $\Sigma^{+}$ & $d$ & $\mathrm{He}^{3}$ & $T^{+}$ & $\alpha$ \\
\hline GARFIELD & $e^{-}$ & $e^{+}$ & $\mu^{-}$ & $\mu^{+}$ & $\pi^{-}$ & $\pi^{+}$ & $K^{-}$ & $K^{+}$ & $p$ & $\bar{p}$ & & & $d$ & & & \\
\hline
\end{tabular}

Table 1: Lists of GEANT and GARFIELD particles 
The comparison reveals that some GEANT particles species have no GARFIELD counterparts. Therefore instead of the missing GARFIELD particles, the available particles with similar masses or electric charges are used, i.e. $\mathrm{He}^{3}$, triton, $\alpha$ are replaced with deuteron and $\Sigma^{-}, \Sigma^{+}$hyperons are substituted by $p, \bar{p}$ respectively.

In the next step the typical number of electrons/ions produced in cluster is estimated. The number of electrons/ions per cluster is random quantity, therefore it must described by reasonable probability distribution. Fig.8 shows examples of such probability distributions for $e^{-}$and $\pi^{-}$at momentum $250 \mathrm{MeV} / \mathrm{c}$.
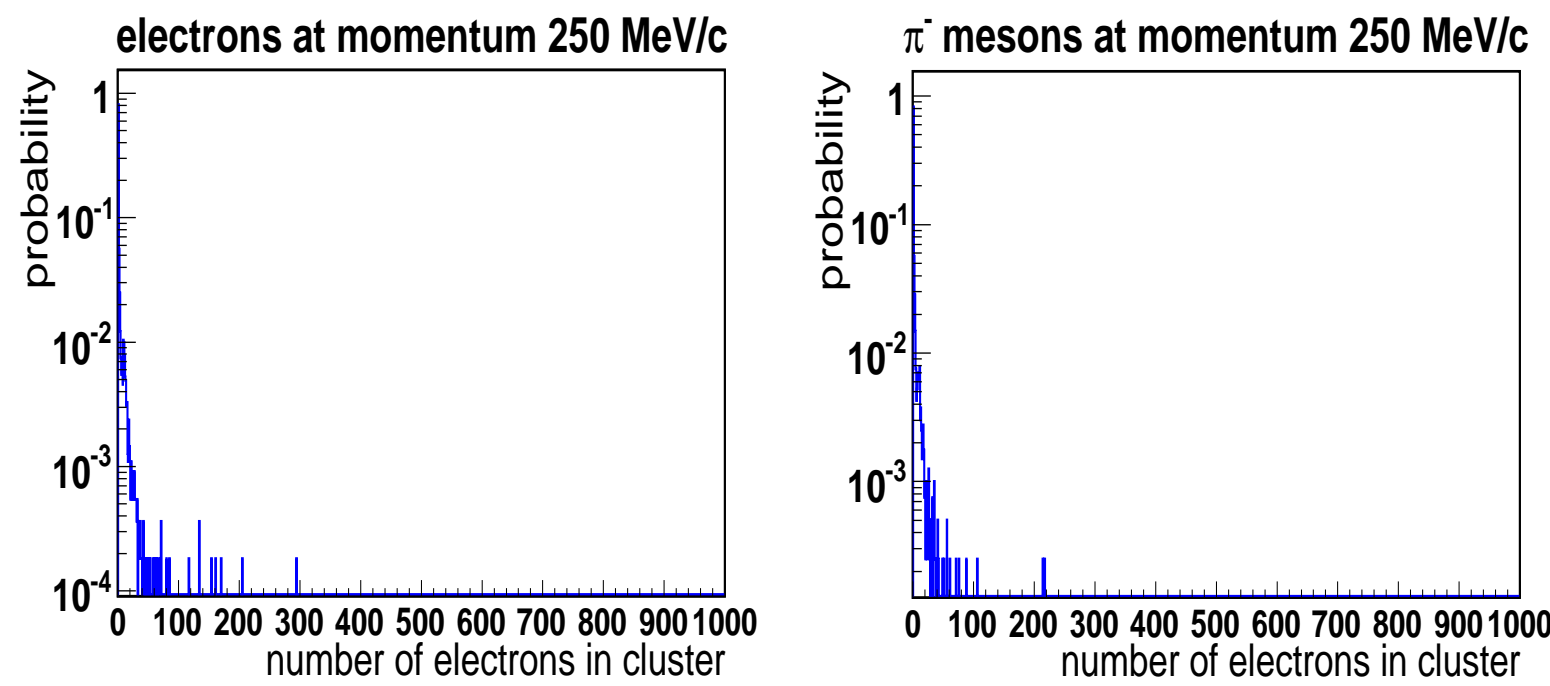

Figure 8: Probabilities to produce cluster with a certain number of electrons/ions

The maximum of the probability spectra is found at 1 electron/ion per cluster and the mean value is about 3 electrons/ions per cluster for all the studied particles. However, as the distributions in Fig.8 suggest, occasionally even large electron/ion clusters may appear, although a likelihood of such events is strongly suppressed.

Fig.9 demonstrates a dependence of number of electrons/ions per cluster on particle momenta. As in the previous Figure, both the examples are shown for $e^{-}$and $\pi^{-}$.

The spectra of most particles under study show dependence only at low momenta (up to 100$150 \mathrm{MeV} / \mathrm{c}$ ) except for $e^{-}, e^{+}$, where a weak dependence is observed only at the lowest momenta (up to $20 \mathrm{MeV} / \mathrm{c}$ ).

Before proceeding to the electron/ion drift simulations, it would be desirable to check if our GARFIELD based simulations of the primary charge are all right. The basic check consists in comparing the GARFIELD primary charges generated in the straw tubes with the corresponding GEANT energy losses.

The conversion of primary charges $q$ to energy losses $\mathrm{d} E / \mathrm{d} x$ is:

$$
-\frac{\mathrm{d} E}{\mathrm{~d} x}=n_{q} E_{\min }
$$

where $n_{q}$ is a number of electrons (ions) with total charge $q$ and $E_{\min }$ is a minimal energy needed to ionize a working gas. For our gas mixture, $E_{\min } \approx 27.5 \mathrm{eV}$. 

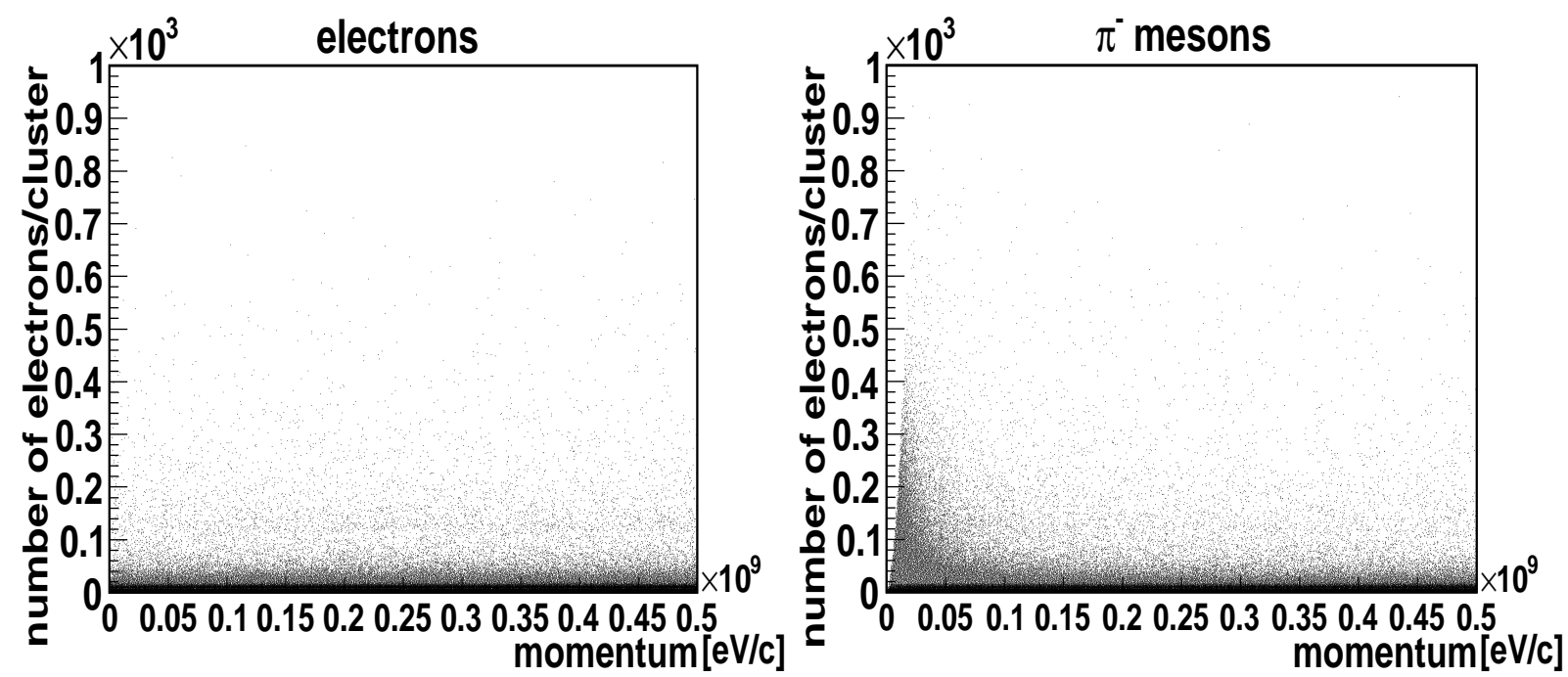

Figure 9: Dependence of number of electrons/ions per cluster on particle momenta - shown spectra for $e^{-}$ and $\pi^{-}$

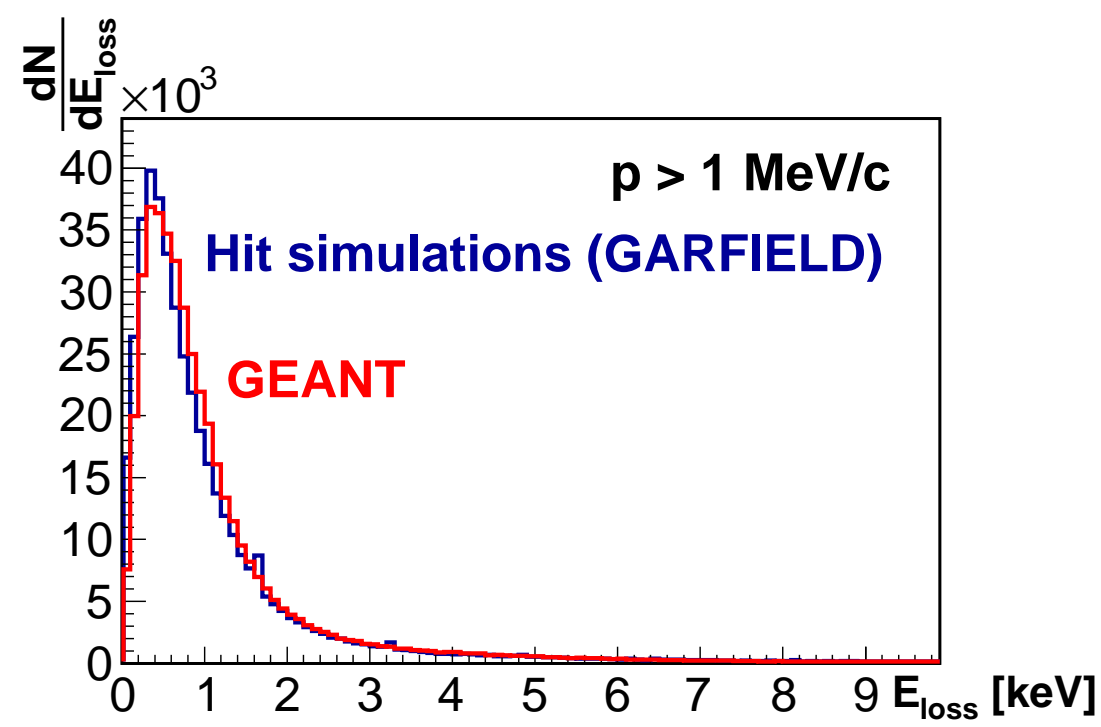

Figure 10: Comparison of GARFIELD and GEANT energy losses

The comparison in Fig.10 proves very good agreement between the GARFIELD and GEANT energy losses, which makes our GARFIELD simulations more credible.

\section{Electron drift, attachment and avalanche simulation}

Drift of primary electrons toward the anode is described by the drift velocity $\mathbf{v}_{\mathbf{d}}$ which is in general a function of electric field $\mathbf{E}$ and magnetic field $\mathbf{B}$

$$
\dot{\mathbf{r}}=\mathbf{v}_{\mathbf{d}}(\mathbf{E}(\mathbf{r}), \mathbf{B}(\mathbf{r}))
$$


The first order equation of motion Eq.6.1 cannot be always analytically solved which results in necessity to adopt a phenomenological approach. There are two phenomenological methods currently implemented in GARFIELD for electron drift simulations. They both includes the processes of transverse and longitudinal diffusion of electrons in gas medium. In the Monte Carlo method Eq.6.1 is stochastically integrated, while the method of microscopic tracking transports electrons on microscopic level, i.e. down to the level of electron mean free path. The microscopic tracking is thus believed to provide more reliable results then the Monte Carlo method whose results may depend on the chosen step of electron drift in radial direction.

Fig.11 demonstrates the dependence of electron drift time on the electron starting radial distance from straw anode wire. The Figure also shows a comparison of the Monte Carlo method with the microscopic tracking.
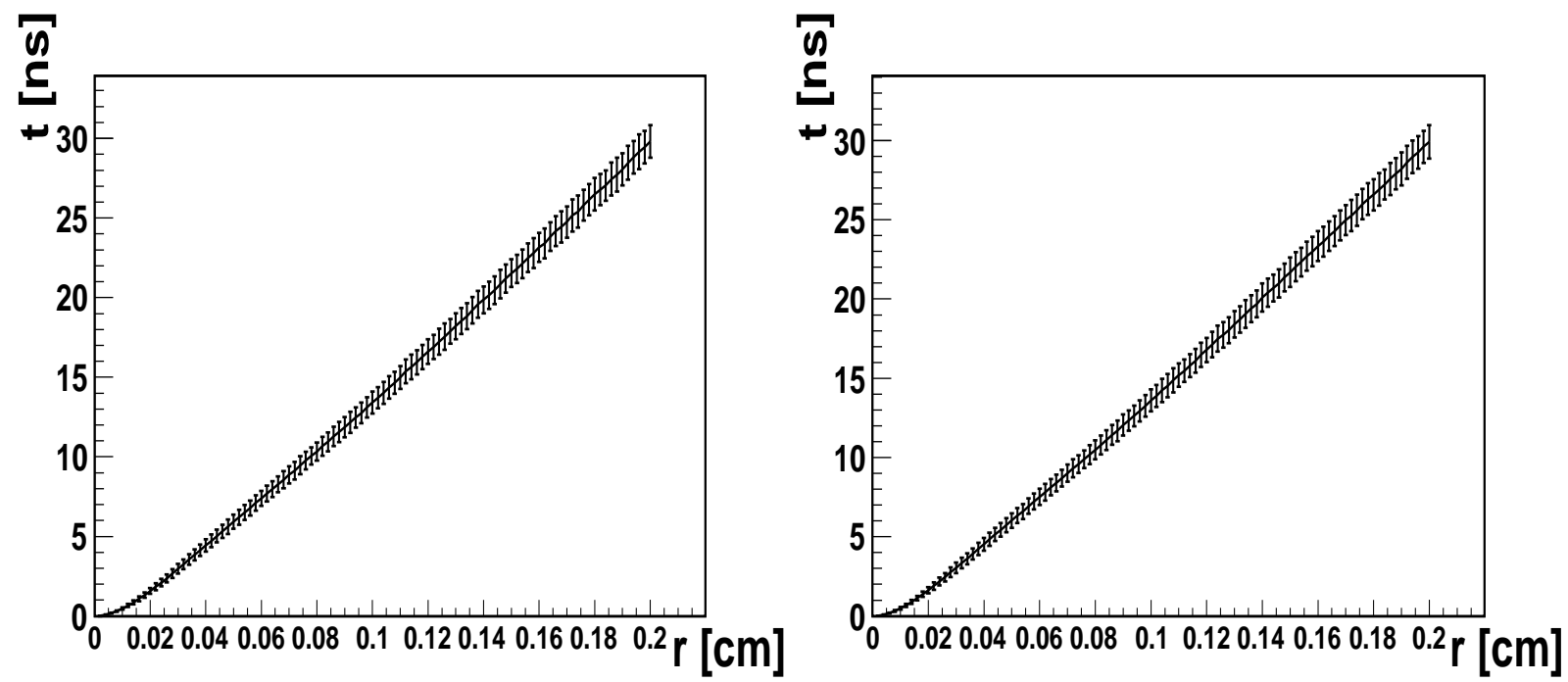

Figure 11: Electron drift time vs radial distance - the MC (left) and the microscopic tracking (right)

There is no obvious difference between the two methods. The maximal electron drift time $t_{\max }^{e^{-}}$ corresponding to the straw tube radius of $2 \mathrm{~mm}$ is about $30 \mathrm{~ns}$. The dependence of the drift time on $r$ seems very close to straight line, except for the avalanche region in the vicinity of anode wire. The error bars reflect fluctuations of the drift time resulting from both the longitudinal and transverse diffusions of electron drift motion. These fluctuations are ultimately responsible for limited resolution of the coordinates estimated by straw detector. Fig. 12 manifests Gaussian behaviour of the drift time fluctuations.

While drifting toward the anode, some electrons are captured by gas molecules inside a tube. The ionization of gas molecules through the attachment of electrons is described by reaction:

$$
A+e^{-} \rightarrow A^{-}
$$

Fig.13(left) shows how the relative amount of captured electrons depends on their starting radial distance $r$ from the straw anode.

Fig.13(left) implies that approximately $20 \%$ of the primary charge is lost if it originates at $r>500 \mu \mathrm{m}$ from anode wire. This behaviour follows directly from the radial dependence of 


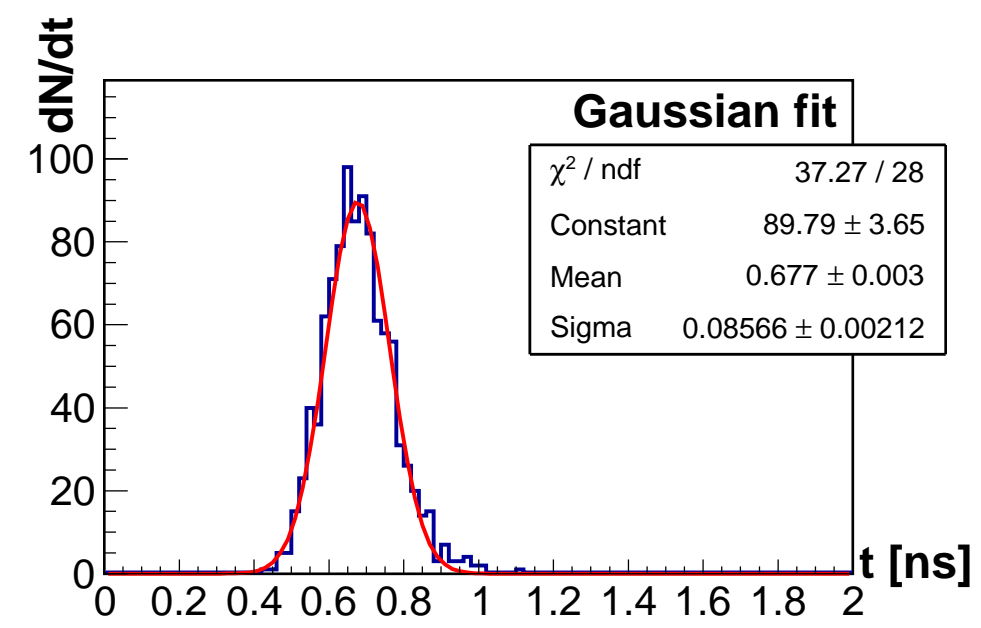

Figure 12: Electron drift time distribution for $r=100 \mu \mathrm{m}$
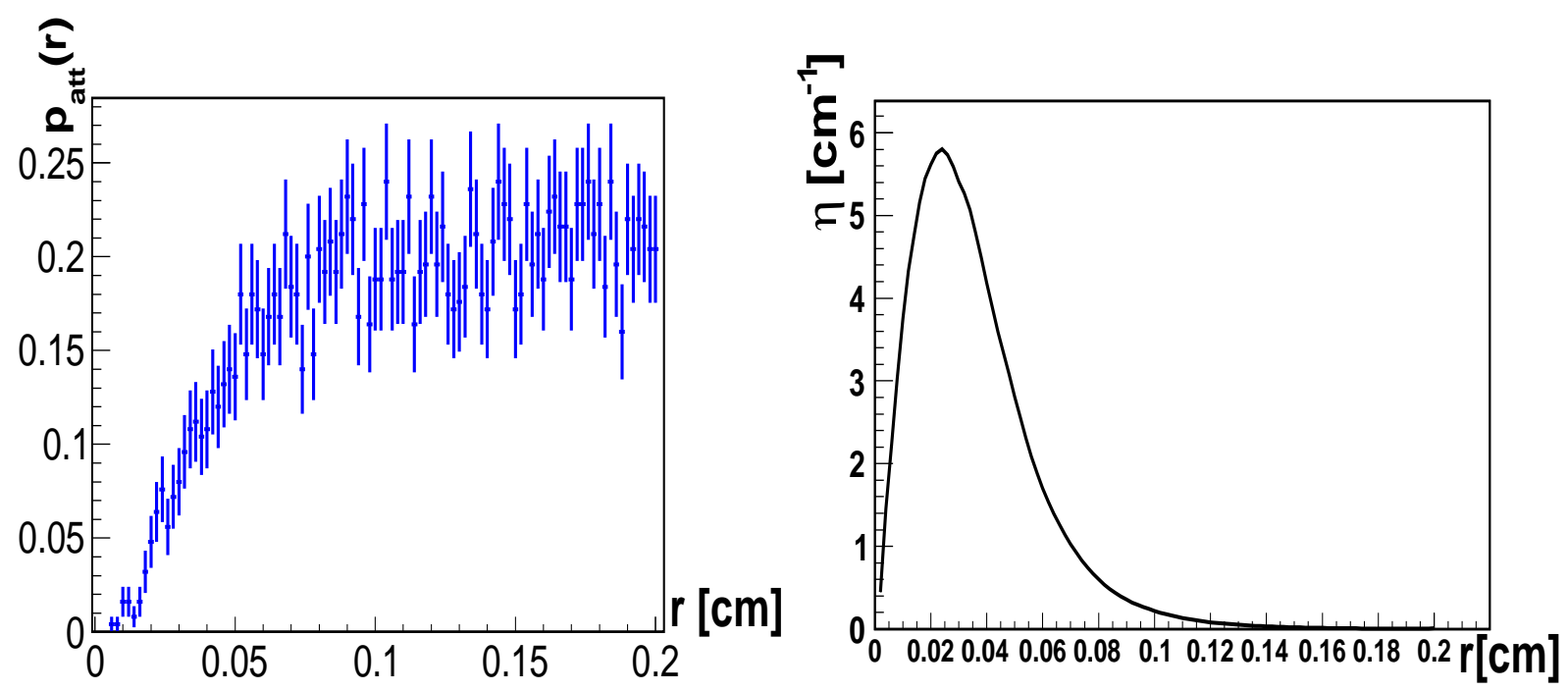

Figure 13: (left) Probability of electron attachment vs radial distance. (right) Radial dependence of attachment coefficient $\eta$

the attachment coefficient $\eta$ which is presented in Fig.13(right). The function in Fig.13(right) is actually the transformed dependence of $\eta$ on electric field $\vec{E}(r)$.

The electrons that succeed to drift close enough to the anode without being attached to gas molecules, produce an avalanche which is characterized by the multiplication factor called gas amplification or gas gain. Fig. 14 shows the gas gain as a function of radial distance $r$ of primary electrons from the anode.

The plateau in Fig.14 ranging from $200 \mu \mathrm{m}$ to $2 \mathrm{~mm}$ suggests the size and placement of the tube drift region while the steep drop below $200 \mu \mathrm{m}$ approximately coincides with the avalanche region. When primary electrons originate in the avalanche region, their gas amplification is lower, since in such a case due to their proximity to the anode an avalanche induced by them does not get evolved to full extent. A constant fit of the plateau region provides mean gas gain about $3 \times 10^{4}$. 


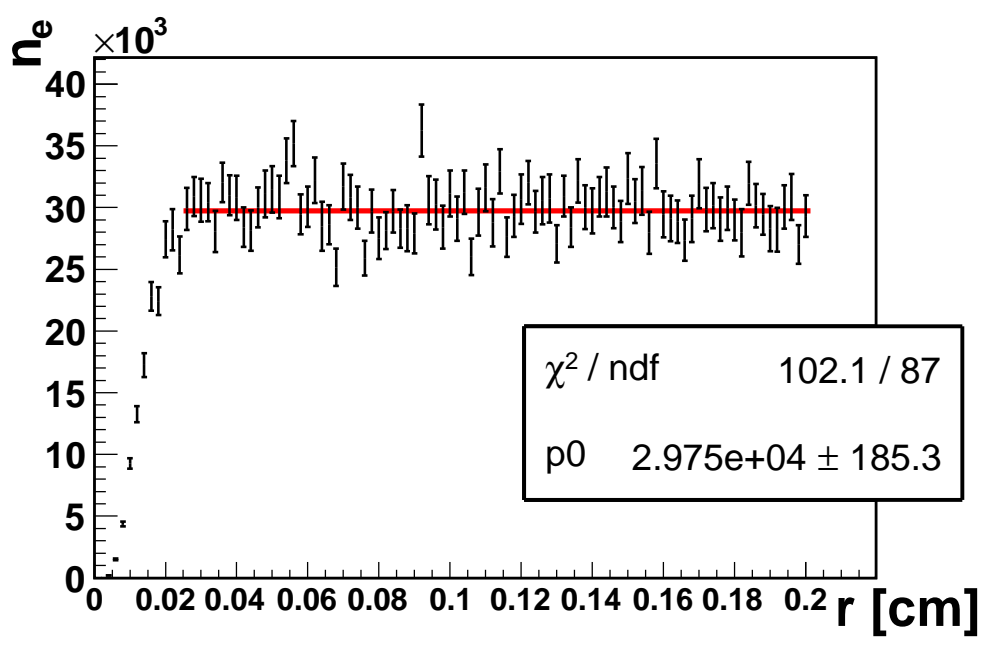

Figure 14: Mean number of avalanche electrons vs radial distance

The fluctuations of gas gain $G$ are described by Polya distribution:

$$
p_{\theta}(G)=\frac{1}{G_{0}} \frac{(\theta+1)^{(\theta+1)}}{\Gamma(\theta+1)}\left(\frac{G}{G_{0}}\right)^{\theta} \exp \left(-(\theta+1) \frac{G}{G_{0}}\right),
$$

where parameter $\theta$ is defined as

$$
\theta+1=\frac{G_{0}^{2}}{\sigma_{G}^{2}}
$$

and $G_{0}, \sigma_{G}$ are a mean gas gain and a standard deviation respectively.

An example of Polya distribution for value of $\theta$ typically seen in our data is presented in Fig.15.

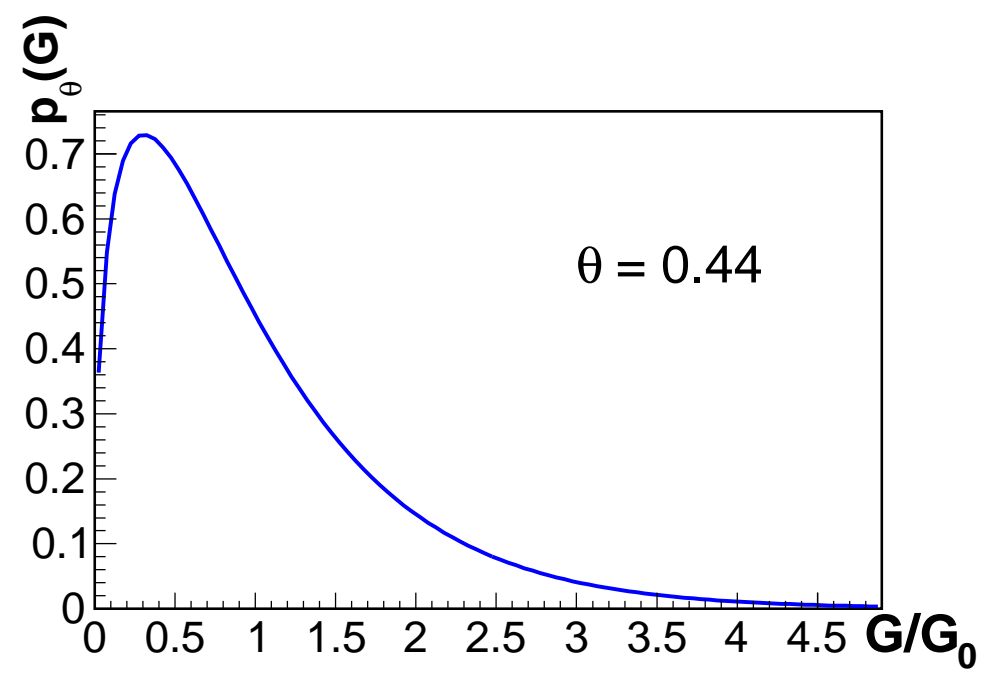

Figure 15: Polya function

The plot confirms that even comparatively large deviations from the mean value $G / G_{0}=1$ of the relative gas gain may occur with still significant probabilities. 


\section{Anode signals}

Signals induced in read-out electrodes by moving charge carriers are calculated applying Ramo-Shockley theorem [7],[8]. Providing the signals are read-out by anodes, a particle with charge $q$ moving at instantaneous velocity $\vec{v}$ at a radial distance $r$ from the anode induces electric current

$$
i_{\text {ind }}(t)=-q \vec{v} \cdot \vec{E}_{w}(\vec{r})
$$

where $\vec{E}_{w}(\vec{r})$ is a so-called weighting field, i.e. produced by unit potential applied to the anode and zero potential applied to the cathode.

If charge $q$ moves from point $x_{0}$ to point $x_{1}$, the induced charge is:

$$
Q_{\text {ind }}=\frac{q}{V_{w}}\left[\Psi\left(x_{1}\right)-\Psi\left(x_{0}\right)\right],
$$

where $V_{w}$ is an anode voltage and $\Psi(x)$ is a potential of the weighting field. Eq.7.2 is just a different form of Ramo-Shockley formula 7.1.

When all the ions and electrons reach the corresponding electrodes, the charge $Q_{\text {electrons }}$ induced by electrons is:

$$
Q_{\text {electrons }}=q_{\text {electrons }}\left[1-\frac{\Psi\left(x_{0}\right)}{V_{w}}\right],
$$

where $q_{\text {electrons }}$ is a full charge of electrons produced in the working gas volume.

On the other hand, the charge $Q_{i o n s}$ induced by motion of ions is

$$
Q_{\text {ions }}=q_{\text {electrons }} \frac{\Psi\left(x_{0}\right)}{V_{w}} .
$$

Using Equations 7.3 and 7.4, it is easy to show the total induced anode charge $Q_{\text {ind }}$ equals $q_{\text {electrons }}$ :

$$
Q_{\text {ind }}=Q_{\text {electrons }}+Q_{\text {ions }}=q_{\text {electrons }} .
$$

In addition to that, Equations 7.3 and 7.4 allow us to compare the contributions from electrons and ions to the total anode charge. In general $Q_{\text {electrons }} \neq Q_{\text {ions }}$. For instance, if $x_{0}$ converges to the anode wire radius $r_{\text {min }}$, then $\Psi\left(x_{0}\right) \rightarrow V_{w}$. Consequently

$$
Q_{\text {electrons }} \rightarrow 0 \quad \text { and } \quad Q_{\text {ions }} \rightarrow q_{\text {electrons }} .
$$

This means that most of the induced anode charge comes from ions.

The previous considerations point out that eventually our attention must be turned to ions. Fig. 16 displays how the drift time of ions inside the straw tube depends on the radial path $r_{\text {max }}-r$ they must traverse while drifting to the cathode with radius $r_{\max }$.

The maximal ion drift time is roughly $40 \mu$ s which is about thousand times larger than the maximal electron drift time (see Fig.11). The difference in the electron and ion drift times is reflected in the different shapes of their anode induced signals as well. Figure 17 presents a comparison of the anode currents induced by the secondary electrons and ions produced from primary electron/ion cluster. 


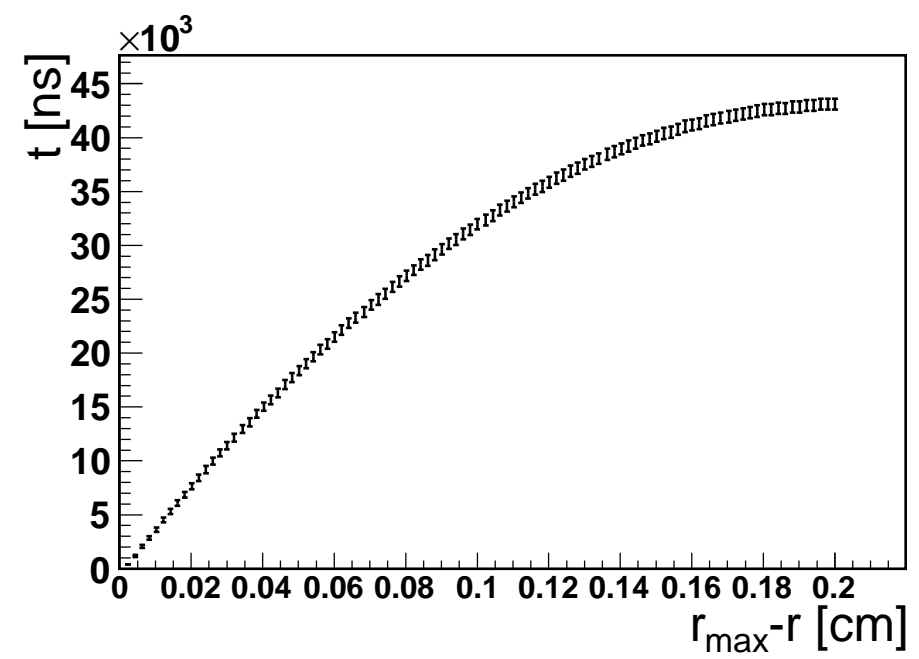

Figure 16: Ion drift time vs complementary radial distance $r_{\max }-r$, where $r_{\max }$ is a tube radius.

The fast drifting electrons generate spikey short-lived anode signals while the slowly moving ions create long tailed signals with a protracted fall-off. Of course, in reality we could observe only a superposition of both the types of signals since their separation is barely possible.

Usually a traversing charged particle leaves more than one electron/ion cluster in the tube volume which finally results in a multiple superposition of the electron and ion signals. Two examples of such superposition are shown in Fig.18.

The left plot corresponds to a particle crossing the tube volume close to the wire whereas the right plot depicts exactly the opposite case. It is easy to comprehend both the pictures when looking on aux-
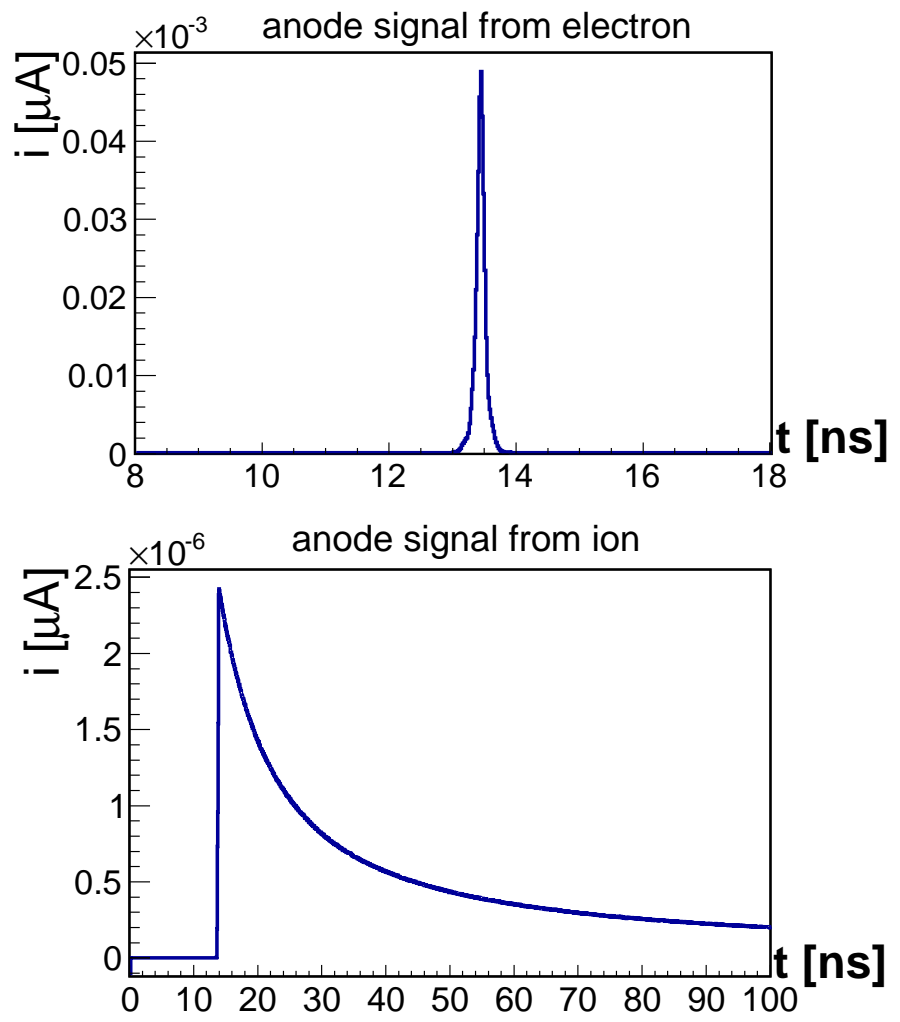

Figure 17: iliary Figure 19.

In the first case the electrons generated from clusters arrive dispersed in time because the drift distances they must travel vary as well. In the second case the electrons originated from different clusters reach the anode almost simultaneously which makes the resulting signal more concentrated and thereby more regular. 

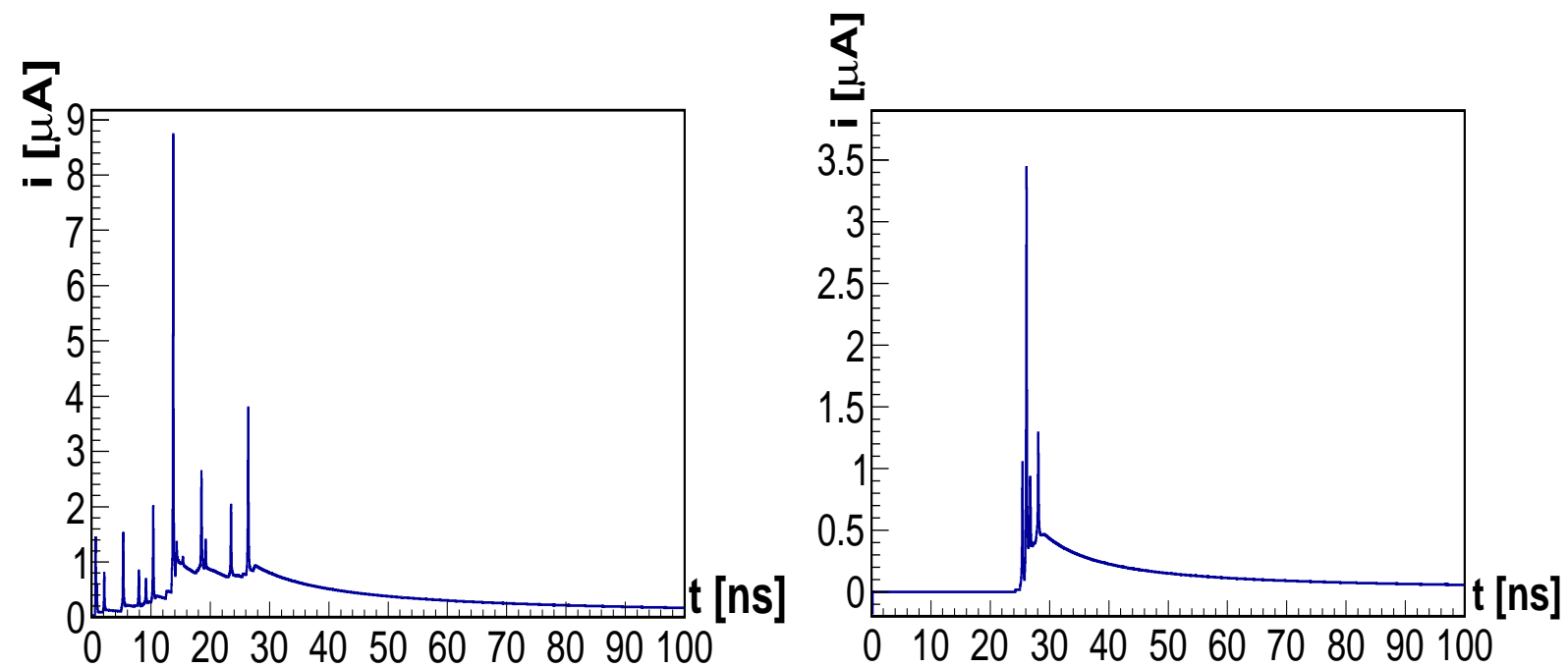

Figure 18: Anode currents stimulated by the particles close to the wire (left) and far from the wire (right)

The shape of the anode signal has an impact on how precisely we are able to estimate the signal onset identified with the minimal drift time. It is obviously more difficult to find an onset of the anode signal for close to wire particles, providing in reality some threshold must be introduced to remove an electronic noise. The details of the discrimination technique employed for estimating the drift time are described in the next section.

In real experiment the anode current spectra analogous to those in Figures 17 or 18 are not available. Instead, we are constrained to work with their integrals, i.e. with the collected anode charges. The time over which the anode current spectra are integrated is often termed a collection

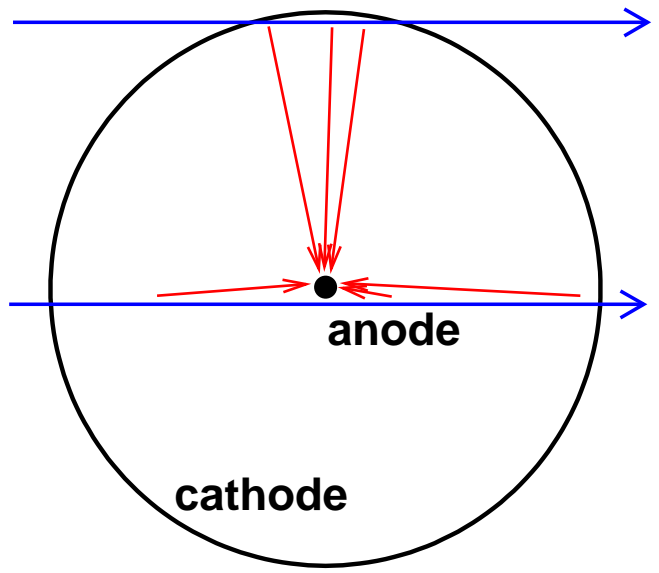

Figure 19: Near and far from the wire particles traversing a straw tube time. The collection time is chosen as a reasonable compromise: On one hand, it should be small enough to ensure a fast detector response, on the other hand, the integrated charge should be sufficiently large to retain information on the total charge left in the tube.

Fig.20 displays a distribution of the anode charge obtained by integration of the anode current over the collection time equal to $60 \mathrm{~ns}$.

As already discussed, the anode charge in Fig.20 is contributed mainly by ions. The contribution from electrons constitutes less than $10 \%$ of the total anode charge.

The anode charge spectra are useful, for instance, to identify abnormal events in the analysed data. 


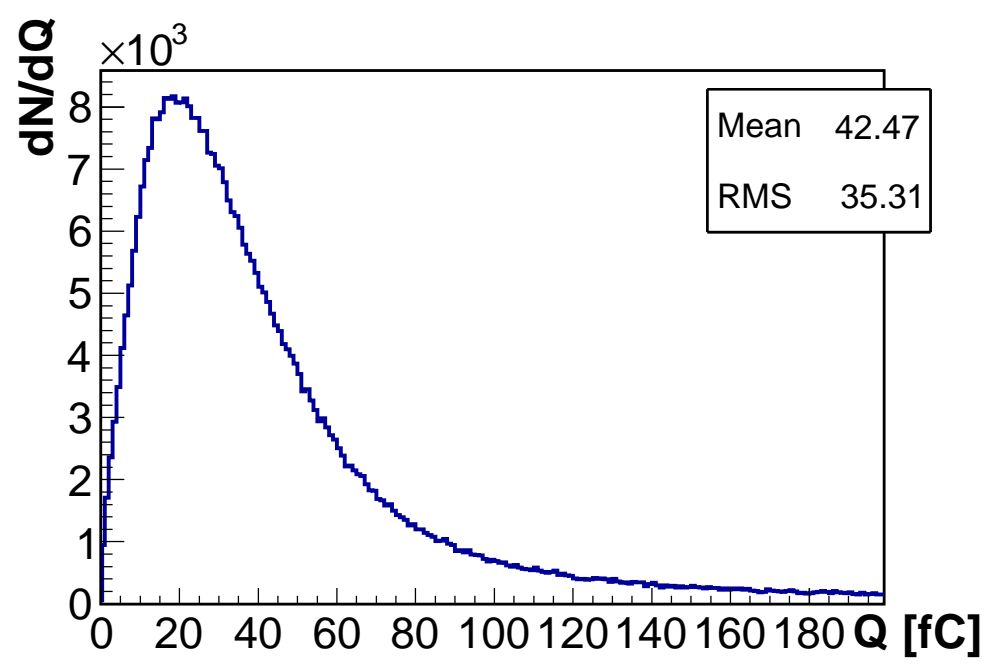

Figure 20: Distribution of the anode charge

\section{Hit coordinates estimation}

Hit coordinates of particles are estimated in terms of DCA - distance of closest approach to the anode wire. We apply the calibration method described in [1], [2] or [9] to determine the DCA coordinates. This approach is applicable under the conditions that the straw tubes are illuminated uniformly and the efficiency is constant over the tube volumes.

The first requirement is tested in Fig.21 showing the distribution of distances of closest approach.

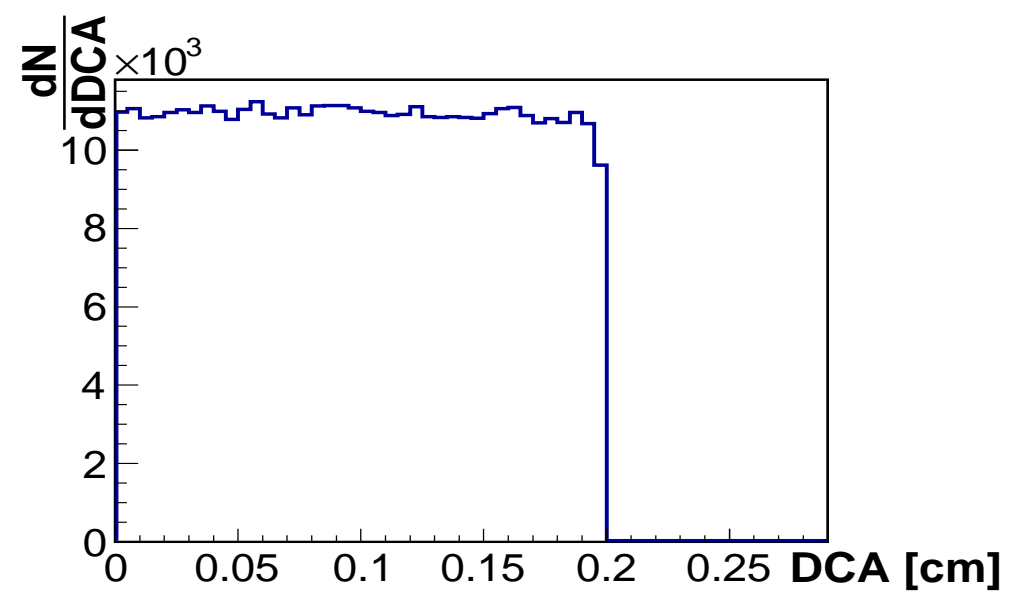

Figure 21: Distance of closest approach

Uniformity of the distribution confirms the straw tubes are irradiated uniformly by particles produced in the analyzed nuclear collisions.

The method uses as input the spectrum $\mathrm{d} N / \mathrm{d} t_{\min }$ of minimal drift times obtained from all the straw tubes in sufficiently large event sample. This spectrum corresponds to experimentally 
measured TDC spectrum. The spectra of minimal drift times used in our calculations are presented in Fig.22.

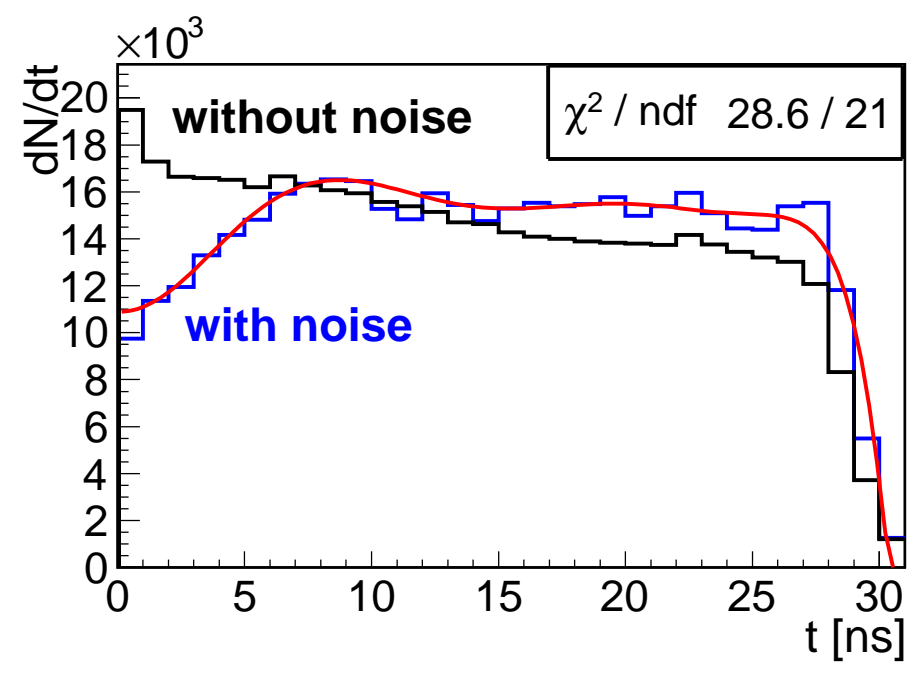

Figure 22: Spectra of minimal drift times

The original drift time spectrum is shown by black solid line. Yet, to allow for the measurement effects, we introduce a noise contribution from the electronic readout system. We add Gaussian constant fraction white noise with an amplitude equal to 3\% of the maximum electric current value. Consequently, the discrimination threshold is set to 5 times higher value to get rid of the noise. The result of this procedure is represented by solid blue line in Fig.22, along with the fit by higherorder polynomial that is shown by red line. The signal delays in the anode wires or in the readout electronics are not yet included in the simulations.

The isochronous radius-time relation displayed in Fig.23 is obtained by multiple integrations of the TDC spectrum:

$$
r(t)=\frac{r_{\max }}{N_{\text {tot }}} \int_{0}^{t} \frac{\mathrm{d} N}{\mathrm{~d} t^{\prime}} d t^{\prime}
$$

gradually increasing time $t$ from 0 to $t_{\max }$ by a predefined step. $N$ in Eq.8.1 is a number of tracks, $r_{\text {max }}$ is the tube radius and $N_{\text {tot }}$ is a total number of tracks.

The radius-time relation is approximated by higher-order polynomial which is used as the calibration curve converting the measured drift times to radial distances from the anode wires.

The estimated radial distances can be compared with the true ones. This comparison is presented in Fig.24(left) in a form of residual distribution. Essentially the plot suggests a resolution of the DCA coordinate.

The standard deviation is almost $300 \mu \mathrm{m}$, however, the HWHM is only $100 \mu \mathrm{m}$. Discrepancy between these two values arises due to the long tails of the residual spectrum which significantly enlarge the standard deviation.

Figure 24(right) demonstrates the dependence of the DCA resolution on radial distance from the anode wire. The plot implies that the DCA resolution varies between 100 and $300 \mu \mathrm{m}$ with the best resolution achieved far from anode wire and the poorest resolution seen close to the wire. The 


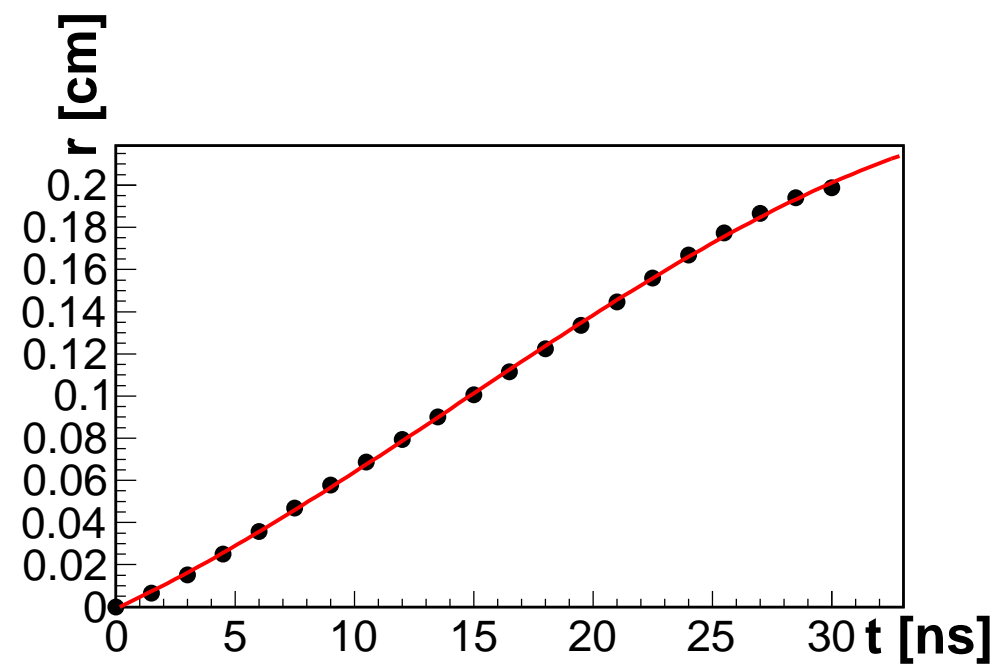

Figure 23: r-t calibration curve
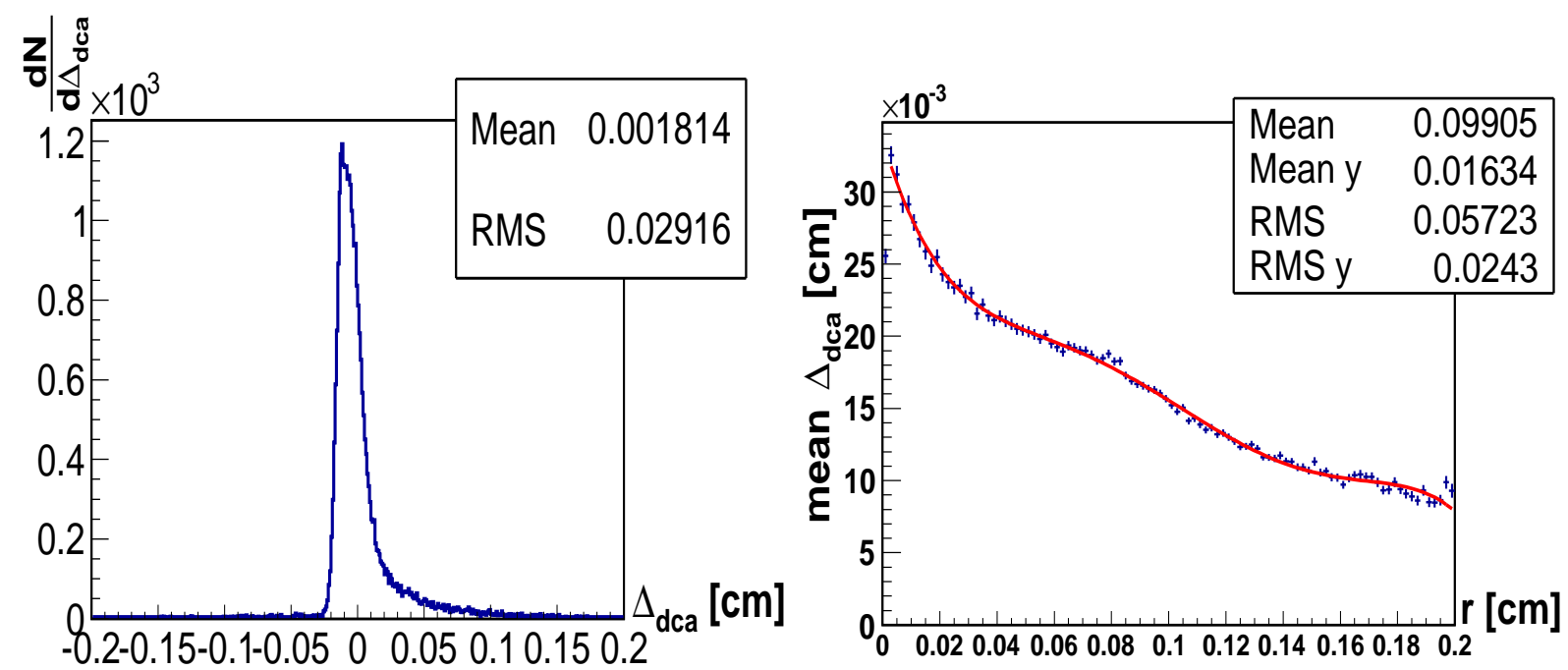

Figure 24: (left) Difference of reconstructed and simulated DCA. (right) Difference of reconstructed and simulated DCA vs radius

similar behaviour is observed in [1],[2],[9] and [10]. The mean DCA resolution is almost $250 \mu \mathrm{m}$ which is somewhat lower than the resolution of $300 \mu \mathrm{m}$ suggested by the previous Figure.

In general the estimated DCA errors are very sensitive to the applied noise and threshold levels. This means that in order to improve the resolution of the hit coordinates measured by the Straw ECT, the noise of readout electronics must be reduced as much as possible.

The hit coordinates are later improved by the so-called autocalibration method [3] which is basically the calibration method applied iteratively in combination with the tracking procedure.

\section{Summary and outlook}

1. The response of the MPD Straw End-Cap Tracker has been estimated employing FAIR- 
ROOT, GEANT3, GARFIELD and the hit simulation and reconstruction programs;

2. The following detector characteristics have been estimated: the occupancy, the charge clusters distributions and energy losses, drift properties (electron and ion drift times, electron attachment probabilities, drift and avalanche regions), gas gain (mean and variation), anode currents, the integrated anode charges;

3. The calibration method has been used to estimate hit distance of closest approach coordinates. The DCA resolution varies from $100 \mu \mathrm{m}$ to $300 \mu \mathrm{m}$ depending on the radial distance from anode wire.

4. The results are found compatible with the results from the other HEP experiments employing straw tube detectors, e.g. PANDA and ATLAS.

5. The obtained results are necessary to proceed to the hit and track reconstruction.

\section{References}

[1] W. Erni et al., PANDA Collaboration, Technical design report for the PANDA (AntiProton Annihilations at Darmstadt) Straw Tube Tracker, Eur. Phys. J. A (2013) 49: 25

[2] G. Avolio et al., Test of the first BIL tracking chamber for the ATLAS muon spectrometer, Nuclear Instruments and Methods in Phys. Res. A 523 (2004) 309-322

[3] A. Biscossa et al., Calypso: a full-scale MDT prototype for the ATLAS muon spectrometer, Nucl. Instr. and Meth. in Phys. Res. A 419 (1998) 331-335

[4] MPD Collaboration, The MultiPurpose Detector - MPD - to study Heavy Ion Collisions at NICA, (Conceptual Design Report),

http://nica.jinr.ru/files/CDR_MPD/MPD_CDR_en.pdf, Dubna, (2008)

[5] R. Veenhof, Garfield - simulation of gaseous detectors, http://garfield.web.cern.ch/garfield/

[6] H. Schindler, Garfield++ - simulation of tracking detectors, http: / / cern. ch/garfieldpp

[7] W. Shockley, Currents to Conductors Induced by a Moving Point Charge, Journal of Applied Physics 9 (1938) 635

[8] S. Ramo, Currents Induced by Electron Motion, Proceedings of the IRE 27 (1939) 584

[9] M. Bellomo et al., The barrel-inner-large tracking chambers for the ATLAS muon spectrometer: Ready for installation, Nucl. Instr. and Meth. in Phys. Res. A 573 (2007) 340-360

[10] V. Peshekhonov et al., Straw Tube Subsystem of the CBM Muon Detector, Physics of Particles and Nuclei Letters 9 (2), (2012) 172-179 